\title{
Space Charge Stopband Correction
}

\author{
Xiaobiao Huang ${ }^{1,2 *}$, S.Y. Lee ${ }^{2}$ \\ ${ }^{1}$ Fermi National Accelerator Laboratory, Box 500, Batavia, IL 60510 \\ 2 Department of Physics, Indiana University, Bloomington, IN 47405
}

September 16, 2005

\begin{abstract}
It is speculated that the space charge effect cause beam emittance growth through the resonant envelope oscillation. Based on this theory, we propose an approach, called space charge stopband correction, to reduce such emittance growth by compensation of the half-integer stopband width of the resonant oscillation. It is illustrated with the Fermilab Booster model.
\end{abstract}

\section{Introduction}

It is well known that the space charge effect causes emittance growth in low energy synchrotrons, such as the Fermilab Booster. We have observed such growth experimentally on the Booster [2]. Fig. 1 shows the normalized vertical emittance of the Booster in the first 4000 turns for beam intensities corresponding to 2-turn to 18-turn injection ( Every 1 turn injection roughly corresponds to $0.5 \mathrm{E} 12$ protons into the Booster from the Linac.). In Fig. 1, two emittance growth patterns are observed. For intensity above 13-turn injection, emittance blows up quickly in the first few hundreds of turns until it reaches a very high level. For intensity below 12turn injection, the emittance growth is more moderate. It has been demonstrated that the latter case can be described by a model which assumes the instantaneous growth rate is composed of a linear term and a space charge term [2]. The space charge term is proportional to the general space charge perveance parameter $K_{\mathrm{sc}}$ defined as

$$
K_{\mathrm{sc}}=2 N r_{0} /\left(\beta^{2} \gamma^{3}\right),
$$

where $N$ is number of protons per unit length and $r_{0}=1.5347 \times 10^{-18} \mathrm{~m}$.

The emittance grows fast for low-energy and high-intensity beams, which is a clear sign of space charge effect. This process can now be simulated with several

*xiahuang@fnal.gov or xiahuang@indiana.edu 


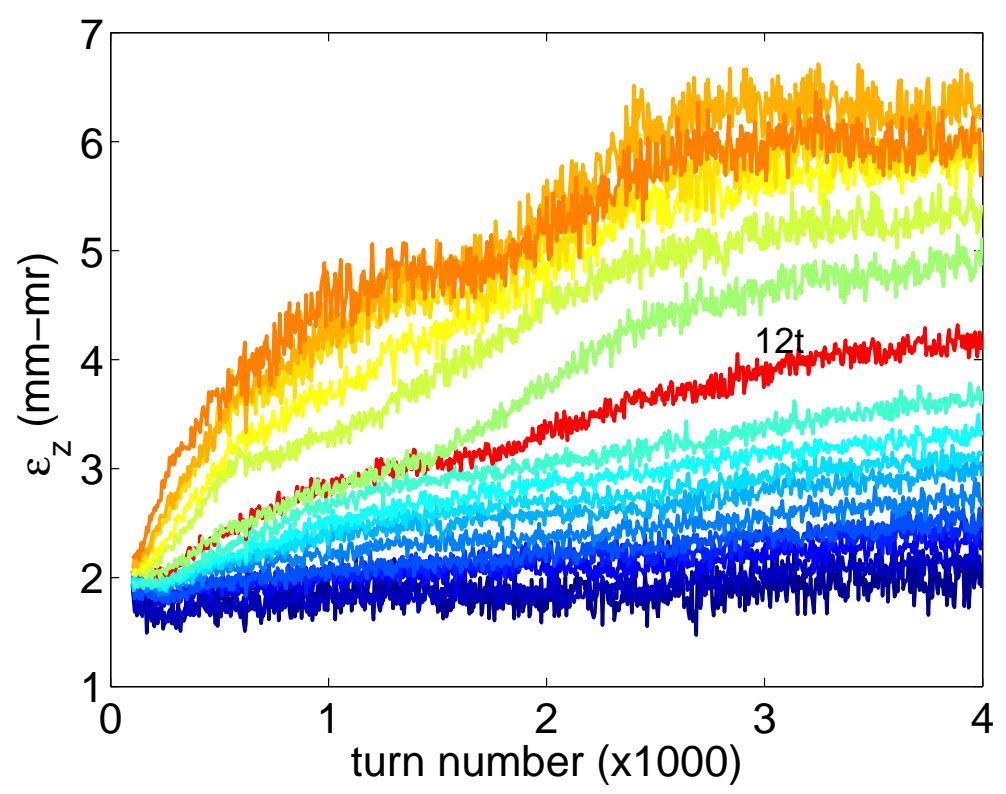

Figure 1: (Color) The normalized vertical rms emittance from 70 turn to 4000 turn for all data sets with 2-turn injection to 18-turn injection. Note the RED curve is for 12-turn injection which marks the border of two kinds of emittance growth behavior.

tracking codes which use the particle-in-cell (PIC) model. However it is still not understood theoretically. Ref [1] suggests a possible mechanism of the space charge induced emittance growth. It relates emittance growth to resonant excitations of the envelope oscillation of mismatched beam. Based on this model, we speculate that it would reduce the emittance growth if we adjust the lattice model to cancel the resonant envelope stopband integral. This approach is called space charge stopband correction.

In this note we describe a method to correct the resonant stopband integrals and demonstrate it with the Fermilab Booster model. We first briefly summarize the theory of resonant envelope oscillation and stopband integrals as in Ref [1].

\section{Envelope oscillation and half-integer stopband}

We use a KV beam to illustrate the envelope oscillation theory. The envelope equation of a $\mathrm{KV}$ beam is given by

$$
R_{\mathrm{b}}^{\prime \prime}+k(s) R_{\mathrm{b}}-\frac{\epsilon^{2}}{R_{\mathrm{b}}^{3}}-\frac{K_{\mathrm{sc}}}{R_{\mathrm{b}}}=0
$$

where $k(s)$ is the focusing function, $\epsilon$ is beam emittance, $R_{\mathrm{b}}$ is the beam envelope radius $\left(R_{\mathrm{b}}=\sqrt{\beta(s) \epsilon}\right.$ for matched beam), $K_{\mathrm{sc}}$ is the space charge perveance parameter and $\beta(s)$ is the betatron amplitude function. Making Floquet transformation 
with

$$
R=\frac{R_{\mathrm{b}}}{\sqrt{\beta(s) \epsilon}}, \quad \phi=\frac{1}{\nu} \int_{0}^{s} \frac{d s}{\beta(s)}
$$

where $\nu$ is the betatron tune, we transform the envelope equation to

$$
\ddot{R}+\nu^{2} R-\frac{\nu^{2}}{R^{3}}-\frac{\nu^{2} \beta(s) K_{\mathrm{sc}}}{\epsilon R}=0
$$

with new time-coordinate $\phi$.

Now we consider the space charge term as a small perturbation. We can expand the actual envelope radius around its unperturbed counterpart to $R=1+r+\Delta$ with a static ( $\phi$-independent) term $\Delta$ and an oscillatory ( $\phi$-dependent) term $r$. Expanding the space-charge factor

$$
\frac{\nu \beta(s) K_{\mathrm{sc}}}{2 \epsilon}=\xi_{\mathrm{sc}}\left(1+\sum_{n=1}^{\infty} q_{n} \cos \left(n \phi+\chi_{n}\right)\right)
$$

into Fourier series, with

$$
\begin{aligned}
\xi_{\mathrm{sc}} & =\frac{1}{2 \pi} \oint \frac{\nu \beta K_{\mathrm{sc}}}{2 \epsilon} d \phi=\frac{K_{\mathrm{sc}} C}{4 \pi \epsilon}, \\
\xi_{\mathrm{sc}} q_{n} & =\frac{1}{\pi} \oint \frac{\nu \beta K_{\mathrm{sc}}}{2 \epsilon} \cos \left(n \phi+\chi_{n}\right) d \phi
\end{aligned}
$$

where $C$ is the circumference of the ring. Inserting the Fourier expanded factor into the envelope equation, we get $\Delta=\xi_{\mathrm{sc}} / 2 \nu$ and

$$
\ddot{r}+\left(4 \nu^{2}-4 \nu \xi_{\mathrm{sc}}\right) r \approx 2 \nu \xi_{\mathrm{sc}} \sum_{n=1}^{\infty} q_{n} \cos \left(n \phi+\chi_{n}\right) .
$$

Eq. (7) shows that the envelope radius can be resonantly excited by the space charge perturbation if the envelope tune $\nu_{\mathrm{env}}=2 \nu-\xi_{\mathrm{sc}}$ is close to an integer. In that case we get

$$
r \approx \frac{2 \nu \xi_{\mathrm{sc}} q_{n}}{-n^{2}+\left(4 \nu^{2}-4 \nu \xi_{\mathrm{sc}}\right)} \cos \left(n \phi+\chi_{n}\right)
$$

with integer $n \approx \nu_{\text {env }}$.

When the envelope is resonantly excited, the betatron phase space mismatch becomes severe. Some particles can be left out of the beam core which then lead to emittance dilution. An efficient way to reduce the emittance growth is thus to reduce the resonant stopband integral $\xi_{\mathrm{sc}} q_{n}$. 


\section{Stopband correction}

Rewriting Eq. (6) into the form

$$
\begin{aligned}
q_{n} & =\frac{1}{\pi} \frac{K_{\mathrm{sc}}}{2 \epsilon \xi_{\mathrm{sc}}} \oint \cos \left(n \phi+\chi_{n}\right) d s \\
& =\frac{2}{C} \oint \cos \left(n \phi+\chi_{n}\right) d s,
\end{aligned}
$$

we see that the stopband arises only from the non-uniform spatial distribution of betatron phase advance. Since the trim quadrupoles perturb the betatron amplitude function as well as the betatron phase advance around the ring, the stopband integrals can be corrected by properly adjusting the trim quadrupoles.

The perturbation to beta function due to trim quadrupoles is

$$
\frac{\Delta \beta}{\beta}=-\frac{\nu_{0}}{2} \sum_{p} \frac{j_{p}}{\nu_{0}^{2}-(p / 2)^{2}} e^{j p \phi},
$$

where $j_{p}$ is the $p$ 'th half-integer stopband integrals for quadrupoles defined as

$$
j_{p}=\frac{1}{2 \pi} \oint k(s) \beta(s) e^{-j p \phi} d s=\frac{1}{2 \pi} \sum_{i}[k \Delta l]_{i} \beta_{i} e^{-j p \phi_{i}}
$$

and the perturbation to phase advance is

$$
\begin{aligned}
\Delta \phi & =\frac{1}{\nu_{0}} \Delta\left(\int_{0}^{L} \frac{d s}{\beta}\right)=-\frac{1}{\nu_{0}} \int_{0}^{L} \frac{\Delta \beta}{\beta} \frac{d s}{\beta} \\
& =-\int_{0}^{\phi} \frac{\Delta \beta}{\beta} d \phi^{\prime}=\frac{\nu_{0}}{2} \sum_{p} \frac{j_{p}}{\nu_{0}^{2}-(p / 2)^{2}} \int_{0}^{\phi} e^{j p \phi^{\prime}} d \phi^{\prime} \\
& =\frac{\nu_{0}}{2} \sum_{p} \frac{j_{p}}{\nu_{0}^{2}-(p / 2)^{2}} \frac{1}{j p}\left(e^{j p \phi}-1\right) .
\end{aligned}
$$

Eq. (12) is verified by comparing $\Delta \phi$ due to one trim quad with MAD calculation and the equation. The comparison is shown in Fig 2.

Thus the perturbation to the stopband integral $q_{n}$ is

$$
\begin{aligned}
\Delta q_{n} & =-\frac{2 n}{C} \oint \sin \left(n \phi+\chi_{n}\right) \Delta \phi d s \\
& =\frac{n \nu}{2 C} \sum_{p} \frac{j_{p}}{\nu^{2}-(p / 2)^{2}} \frac{1}{p} \oint\left(e^{j\left(n \phi+\chi_{n}\right)}-e^{-j\left(n \phi+\chi_{n}\right)}\right)\left(e^{j p \phi}-1\right) d s \\
& =-\frac{\nu}{2\left(\nu^{2}-(n / 2)^{2}\right)}\left(j_{n} e^{-j \chi_{n}}+j_{-n} e^{j \chi_{n}}\right),
\end{aligned}
$$

where in the last step we keep only terms with $p= \pm n$. For each trim quadrupole, $j_{ \pm n}=\frac{1}{2 \pi}[k \Delta l] \beta e^{\mp j n \phi}$, hence the correction of the resonant stopband integral due to the $i$ 'th trim quadrupole is

$$
\Delta q_{n}^{i}=-\frac{\nu}{\nu^{2}-(n / 2)^{2}} \frac{[k \Delta l]_{i} \beta_{i}}{2 \pi} \cos \left(n \phi_{i}+\chi_{n}\right) .
$$




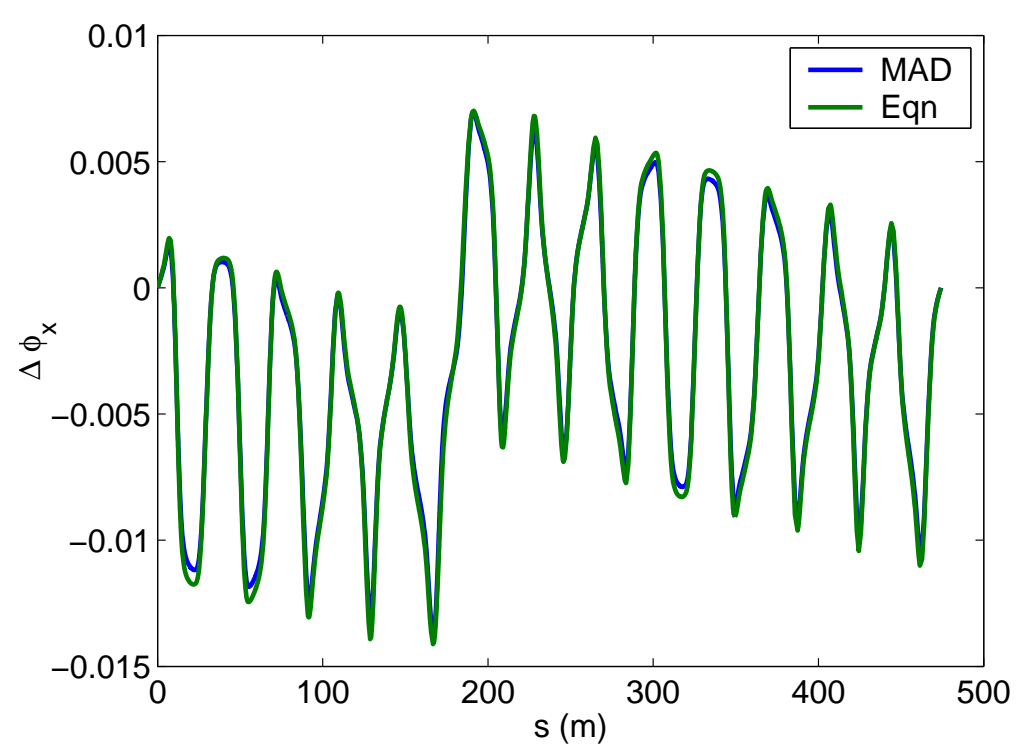

Figure 2: (Color) Comparison of $\Delta \phi_{x}$ due to an increase of QS-9 by $0.5 \mathrm{~A}$ with MAD calculation and Eq. (12) (using 20 harmonics). Eq. (12) is verified.

Eq. (14) is also verified with the Booster lattice. For example, with the operation setting when data in Fig. 1 were taken the model gives $q_{x, 13}=0.1494$ and $\Delta q_{x, 13}^{S 1}=$ 0.0866 for a change of $1.0 \mathrm{~A}$ of trim quadrupole S1. Reducing the current of trim quadrupole $\mathrm{S} 1$ by $0.2 \mathrm{~A}$ and re-evaluating the stopband integral we get $q_{x, 13}=$ 0.1338 . The change of $q_{x, 13}$ is -0.0156 , compared to $0.0866 \times(-0.2)=-0.0173$. For the same setting, $q_{z, 14}=0.1208$ and $\Delta q_{z, 14}^{L 2}=0.0619$. Reducing trim quadrupole L2 by $0.2 \mathrm{~A}$ brings $q_{z, 14}$ down to 0.1079 , which also verifies the equation.

In practice, we want to correct stopband integrals for both the horizontal and vertical planes. And it is desirable to compensate the two harmonics nearest to $\nu_{\text {env }}$. For example, for the Booster, we want to compensate $q_{x, 13}, q_{x, 14}, q_{z, 13}$ and $q_{z, 14}$. A working correction scheme needs to consider all these harmonics. It can be done by defining a merit function which include all 4 harmonics

$$
f=q_{x, 13}^{2}+q_{x, 14}^{2}+q_{z, 13}^{2}+q_{z, 14}^{2}
$$

with

$$
q_{y, n}=q_{y, n 0}+\sum_{i} \Delta q_{n}^{i} \Delta I_{i}
$$

where $y$ stands for $x$ or $z$ and $\Delta I_{i}$ is the change of the $i$ 'th trim quadrupole. Using the gradient of function $f$, it is very easy to reduce it, along with all related harmonics. The stopband correction for the Booster is described in the next section. 

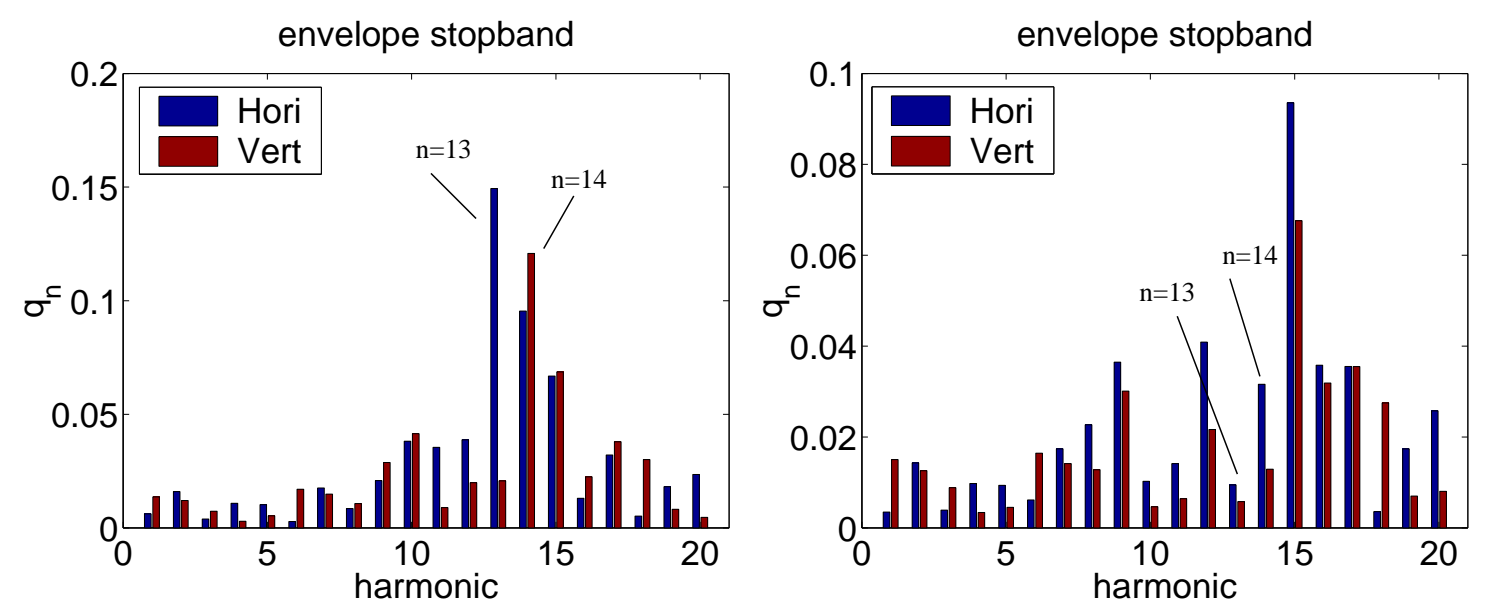

Figure 3: Left: the envelope stopband integrals for the Booster with recorded operation settings. Right: the corrected envelope stopband integrals.

\section{Stopband correction for the Booster}

For the Booster, the nominal betatron tunes are $\nu_{x}=6.7$ and $\nu_{z}=6.8$, which make the 13rd and 14th harmonics most dangerous. Unfortunately, these harmonics are unusually large, as shown in Fig. 3 (left), which shows the stopband integrals with the recorded setting for data in Fig. 1. It is seen that $q_{x, 13}$ and $q_{z, 14}$ are much higher than other harmonics.

Using the stopband correction method described in the last sub-section, we have successfully reduced these stopband integrals to a very low level. The corrected result is shown in Fig. 3 (right) which shows that $q_{x, 13}$ and $q_{z, 14}$ are down to 0.01 . The harmonics before and after correction are also shown in Table 1 . If the envelope oscillation is indeed the cause of emittance growth, such correction of envelope stopband would dramatically improve the beam performance. The correction setting only changes half of the trim quadrupoles by up to $0.4 \mathrm{~A}$.

Table 1: The concerned harmonics before and after correction

\begin{tabular}{|l|l|l|l|l|}
\hline & $q_{x, 13}$ & $q_{x, 14}$ & $q_{z, 13}$ & $q_{z, 14}$ \\
\hline initial & 0.1494 & 0.0954 & 0.0207 & 0.1208 \\
corrected & 0.0095 & 0.0316 & 0.0058 & 0.0129 \\
\hline
\end{tabular}

A big concern in practice is of course the sensitivity of the calculated correction setting. The maximum sensitivity is estimated by

$$
\left|\frac{\Delta q_{n}^{i}}{\Delta I_{i}}\right| \leq\left|\frac{\nu}{\nu^{2}-(n / 2)^{2}} \frac{[k \Delta l]_{i} \beta_{i}}{2 \pi}\right|
$$

according to Eq. (14). Using $\nu_{x}=6.7, k \Delta l=0.0068 \mathrm{~m}^{-1}$ for $1 \mathrm{~A}$ at $400 \mathrm{MeV}$, $\beta_{x}=34.0 \mathrm{~m}$ in average at short sections, we get a maximum change of $q_{x, 13}$ of 
0.094 by 1 A change of current of a short section trim quadrupole. Using $\nu_{z}=6.8$, $\beta_{z}=21.0 \mathrm{~m}$ at long sections in average, the maximum sensitivity is 0.056 by $1 \mathrm{~A}$ change of a long section trim quadrupole. Such sensitivity levels are within the controllable range in experiments. However, one has to have an accurate lattice model to find a meaningful correction setting. We expect that the model should be accurate enough to assure the error of the calculated lattice functions (beta function and phase advance) are within the error level due to $1 \mathrm{~A}$ change of one trim quadrupole. This is beyond the model accuracy we currently have.

We have tried this approach on the Booster but did not obtain positive results. The experiment was conducted as follows. First we took the settings of all trim quadrupoles and built a lattice model (MAD) with them. The stopband integrals and the desired correction setting (of trim quads) were calculated using the model. We then dialed in the correction setting and observed the emittance growth in the beginning of the cycle. We also used the knob to change the currents of a few trim quads around the calculated setting. The negative result can be explained by the fact that the precision of the lattice model is not adequate. To succeed in the future, we may need a better search strategy to locate the working correction setting without a precise lattice model, or we can improve our model precision by calibration with beam-based measurements.

\section{References}

[1] S. Y. Lee, "Accelerator Physics", second edition (World Scientific, Singapore, 2004).

[2] X. Huang and S. Y. Lee, "Emittance Measurements and Modeling of the Fermilab Booster", Fermilab Technote FERMILAB-TM-2326-AD (2005). 